\title{
RELACÃO ENTRE AMBIDESTRALIDADE E SISTEMAS DE CONTROLES GERENCIAIS EM ONGS BRASILEIRAS
}

\author{
Adrinana de Azevedo Ramos Bandeira Arantes ${ }^{1}$ \\ Juliano Lima Soares ${ }^{2}$
}

Resumo:

Objetivo: Baseado em dois construtos, a Ambidestralidade, que preconiza o alcance do equilíbrio das ações de inovações radicais e incrementais, e os Sistemas de Controles Gerenciais, entendidos como um conjunto de esforços com o objetivo de aumentar a probabilidade de que as pessoas tenham comportamentos e objetivos alinhados aos objetivos organizacionais, este artigo tem por objetivo investigar a relação entre o uso de Sistemas de Controles Gerenciais e a Ambidestralidade Organizacional em ONGs Brasileiras.

Metodo: A pesquisa é descritiva com abordagem quantitativa, realizada por meio de survey como instrumento de coleta de dados, utilizando escala do tipo Likert. A amostra da pesquisa compreendeu 227 respostas válidas de um universo de 2.200 unidades da Associação de Pais e Amigos dos Excepcionais (Apae) distribuídas em mais de 2.000 municípios brasileiros. A análise dos dados foi realizada a partir de estatística descritiva, testes de correlação de Pearson e regressão linear múltipla.

Resultados: A literatura tem abordado a relação entre esses dois construtos em organizações privadas, evidenciando relação positiva entre eles. A abordagem neste trabalho, traz como resultados a evidenciação de que em organizações não governamentais esta relação também é positiva e significativa, indicando que, o uso dos Sistemas de Controles Gerenciais pode propiciar um ambiente inovador.

Contribuições: Os resultados encontrados contribuem para a compreensão do uso de Sistemas de Controles Gerenciais em seus vários níveis e suas relações com as ações de inovação com vistas ao desenvolvimento dessas organizações.

Palavras-chave: Ambidestralidade Organizacional, Sistemas de Controles Gerenciais, organizações não governamentais.

${ }^{1}$ contadoraadrianabandeira@gmail.com. Universidade Federal de Goiás. Goiania-GO, Brasil. https://orcid.org/0000-0001-8743-8686

${ }^{2}$ julianolimasoares@ufg.br. Universidade Federal de Goiás. Goiania-GO, Brasil. https://orcid.org/0000-0001-8056-4794

- DOI: http://dx.doi.org/10.14392/asaa.2020130308

- Artigo submetido em: 03/04/2020. Revisöes requeridas em: 11/11/2020. Aceito em: 05/12/2020 


\title{
PERFORMANCE OF CORPORATE ACQUISITION BETWEEN BRAZILIAN FAMILY AND NON-FAMILY PUBLIC FIRMS
}

\begin{abstract}
:
Objective: Based on two constructs, Ambidexterity, which advocates achieving a balance of radical and incremental innovation actions, and Management Control Systems, understood as a set of efforts with the objective of increasing the likelihood that people will behave and objectives aligned with organizational objectives, this article aims to investigate the relationship between the use of Management Control Systems and Organizational Ambidexterity in Brazilian NGOs.

Method:The research is descriptive with a quantitative approach, carried out through a survey as a data collection instrument, using a Likert scale. The research sample comprised 227 valid responses from a universe of 2,200 units of the Association of Parents and Friends of the Exceptional (Apae) distributed in more than 2,000 Brazilian municipalities. Data analysis was performed using descriptive statistics, Pearson's correlation tests and multiple linear regression.

Results: The literature has addressed the relationship between these two constructs in private organizations, showing a positive relationship between them. The approach in this work, results in the evidence that in non-governmental organizations this relationship is also positive and significant, indicating that the use of Management Control Systems can provide an innovative environment. Contributions: The results found contribute to the understanding of the use of Management Control Systems at its various levels and their relationship with innovation actions with a view to the development of these organizations.
\end{abstract}

Keywords: Organizational Ambidexterity, management control systems, non-profit organizations. 


\section{INTRODUÇÃO}

É crescente o número de estudos que argumentam que empresas consideradas ambidestras têm maior possibilidade de obter sucesso em ambientes de mudanças por terem habilidades de explorar simultaneamente as suas competências existentes e adaptar-se explorando novas oportunidades (Lin \& Chang, 2015; Raisch, Birkinshaw, Probst, \& Tushman, 2009). Em seu artigo "Exploration and Exploitation in organizational Learning", March, em 1991, tornou conhecido o conceito de "Ambidestria Organizacional". Uma empresa é considerada ambidestra quando tem equilíbrio entre as habilidades de inovação incremental: exploitation e de inovação radical: exploration (Dunkan, 1976; Tushman \& O'Reilly , 1996).

Para Jansen, Bosch e Volberda (2006), exploitation é essencialmente o refinamento e a extensão das competências, tecnologias e paradigmas existentes. Seus retornos são positivos, imediatos e previsíveis. Exploration, por sua vez, é fundamentada na experimentação de novas alternativas, seus retornos são incertos, distantes e muitas vezes negativos.

Para se manter sustentável a empresa precisa mostrar vantagem competitiva. Corroborando com esse entendimento, Probst e Raisch (2005) argumentam que um dos grandes motivos para o enfraquecimento competitivo das organizações é a incapacidade de incluir em suas estratégias habilidades para inovar e mudar.

Para assegurar que a estratégia estabelecida pela organização seja colocada em prática e que os objetivos definidos sejam alcançados de forma eficiente e eficaz, com possibilidade de aprimorá-los quando necessário, os gestores se utilizam dos Sistemas de Controles Gerenciais (Beltrami, Gomes, \& Araújo, 2013). Simons (1990) abordou os Sistemas de Controle Gerencial como procedimentos e rotinas formalizadas que usam informações para manter ou alterar padrões nas atividades organizacionais. Flamholtz (1996) acrescenta que o objetivo final de um sistema de controle não é controlar o comportamento específico das pessoas, mas influenciar as pessoas a se comportarem e tomarem decisões que sejam consistentes com os objetivos organizacionais.

Os controles gerenciais oferecem aos gestores informações relevantes à tomada de decisões. Eles também são parte de um processo em constante estado de mudanças e exercem influência positiva sobre a gestão da inovação (Simons, 1990). Nesse mesmo entendimento, Beuren e Oro (2014) destacam que os controles formais subsidiam o processo de gestão da inovação ao fornecer informações. Isso implica em alinhamento do uso de instrumentos do Sistema de Controle Gerencial com o nível de inovação em organizações, de forma a envolver a organização no reconhecimento de incertezas com o objetivo de criar e não evitar as mudanças necessárias para a inovação (Davila, Foster, \& Oyon, 2009).

Este estudo pretende investigar a relação entre Ambidestralidade e Sistemas de Controles Gerenciais em organizações não governamentais brasileiras. No ambiente das organizações sem fins lucrativos, atualmente verifica-se que a motivação ético-social e o profissionalismo técnico não são suficientes, uma vez que tais organizações precisam avaliar como os recursos financeiros e não financeiros limitados podem ser utilizados de maneira eficiente e eficaz mantendo-se sustentáveis (Teelken, 2008; Ramadan \& Borgonovi, 2015). Dessa forma, a mensuração dos resultados é considerada essencial no setor sem fins lucrativos (Julian \& Clapp, 2000; Moxham, 2009; Costa, Ramus, \& Andreaus, 2011).

O estudo é construído a partir de amostra por adesão das Associações de Pais e Amigos dos Excepcionais - APAE - distribuídas em 2.200 unidades, estando presentes em mais de 2.000 municípios brasileiros, 
representando de forma consistente as organizações não governamentais brasileiras em razão de sua consolidação e capilaridade.

A contribuição teórica deste trabalho ocorre pela evidenciação do uso de Sistemas de Controles Gerenciais por organizações não governamentais e sua contribuição com estratégias inovativas no setor estudado, destacando competências de exploitation e exploration, além de demonstrar o nível de Ambidestralidade Organizacional nesse ambiente. A contribuição prática deste estudo para o ambiente de trabalho se dá pela apresentação de um panorama em organizações não governamentais do uso de Sistemas de Controles Gerencias e o grau de Ambidestralidade das organizações, contribuindo com o aprimoramento dos mecanismos de controle, com o aperfeiçoamento de políticas de inovação.

Este artigo está organizado em seis seções, incluindo esta introdução. Na seção 2 é apresentada a fundamentação teórica; a seção 3 inclui os aspectos metodológicos; na seção 4 os resultados são apresentados e discutidos; as discussões são apresentadas na seção 5 e os comentários finais são fornecidos na seção 6.

\section{REFERENCIAL TEÓRICO}

\subsection{Sistemas de Controles Gerenciais e Ambidestralidade Organizacional}

Um Sistema de Controle Gerencial é compreendido como um conjunto de mecanismos, processos e técnicas com o objetivo de aumentar a probabilidade de as pessoas se comportarem de maneiras que levem à consecução dos objetivos organizacionais (Abernethy \& Chua, 1996; Flamholtz, 1996; Beltrami, Gomes e Araújo, 2013). Neste mesmo sentido, o estudo de Bedford, Bisbe \& Swenney (2019) mostra que gerentes usam as medidas contábeis e de desempenho para incentivar a troca de informações dentro da alta gestão em empresas ambidestras para influenciar comportamentos de subordinados.

Neste contexto destaca-se que a relevância da informação contábil está na forma como os gestores a utilizam e interpretam por meio de comunicações com as interações organizacionais (Hall, 2010). Desta forma, para se tornar tangível um Sistema de Controle Gerencial faz-se uso de modelos que possam ser utilizados para descrever uma estrutura (Flamholtz, 1996). Pesquisas recentes (Bedford, 2015; Bedford, Malmi, \& Sandelin, 2016; Frezatti, Bido, Cruz \& Machado 2017) abordam diversos modelos, pois os Sistemas de Controles Gerenciais não são plenamente apropriados para todas as empresas e o mesmo modelo não deve ser aplicado a todas as empresas e em todos os cenários (Merchant \& Van der Stede, 2007; Otley, 1980). Os modelos abordados de maneira significativa pela literatura, relacionando o Sistema de Controle Gerencial e inovação, são os modelos de Simons (1995) e de Malmi e Brown (2008). Tais modelos são base para os modelos abordados tanto na sequência do referencial teórico como no instrumento de coleta.

A abordagem de modelo teórico abrangente é denominada Levers of Control, na qual as tensões dinâmicas encontram-se em equilíbrio possibilitado pela presença de controles positivos e negativos, equilíbrio este responsável por garantir a eficácia do controle de forma variável, de acordo com cada organização. Os controles positivos motivam, contemplam, guiam e favorecem a aprendizagem. Já os controles negativos são responsáveis por limitar, determinar e controlar (Simons, 1995; Kruis, Speklé, \&Widener, 2016; Pletsch, Lavarda, \& Lavarda, 2016). A estrutura desse modelo pode ser visualizada na Figura 1. 
Figura 1: Modelo teórico do Sistema de Controle Estratégico

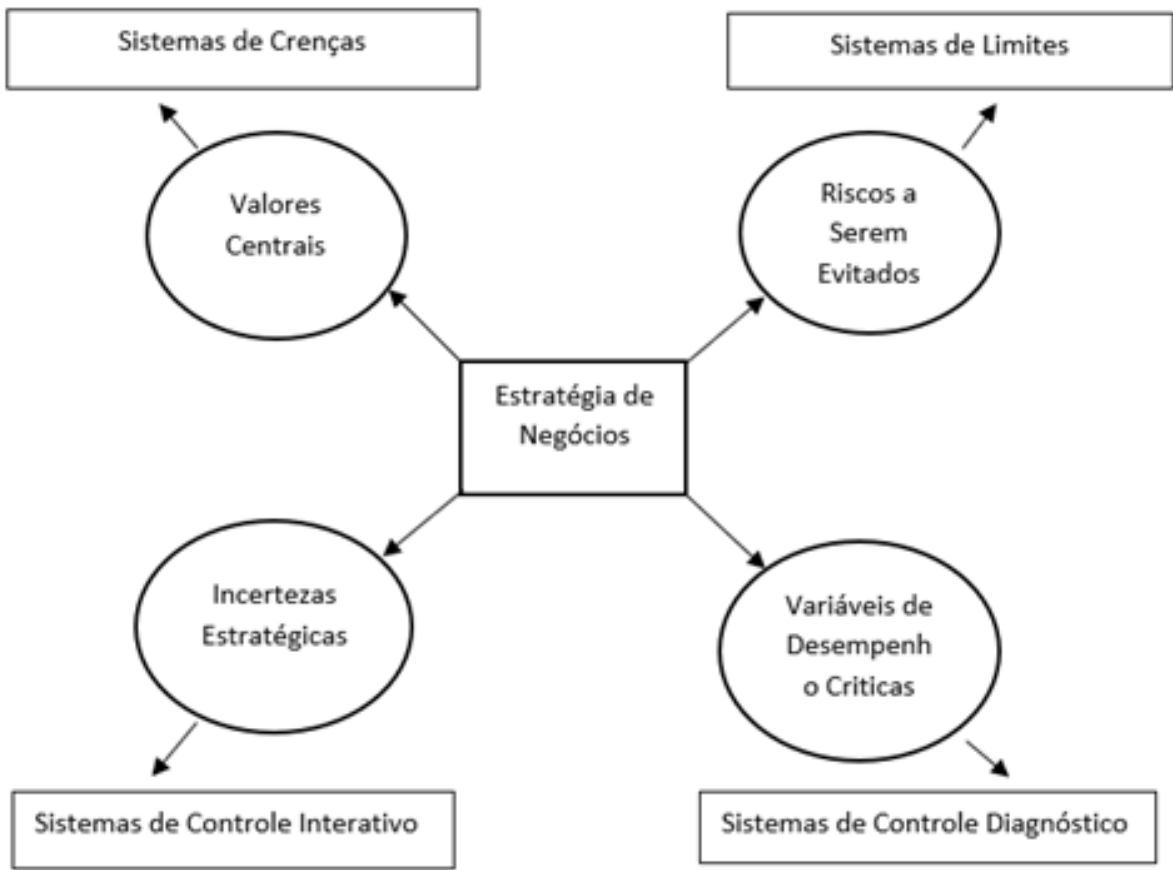

Fonte: De "Levers of Control", de R. Simons, 1995, Harvard Business School Press, Boston.

A abordagem de Malmi \& Brown (2008) para a compreensão dos Sistemas de Controle Gerencial é a de que seus componentes devem ser tratados como um pacote no qual a força da tipologia está no amplo escopo dos controles no Sistema de Controle Gerencial. A visão desse sistema como um pacote adota cinco formas de abordagens de controle, conforme Figura 2: administrativo; cibernético; remuneração e recompensas; planejamento; e cultural (Malmi \& Brown, 2008).

O planejamento é uma forma ex-ante de controle (Flamholtz, Das, Tsui, 1985), definindo os objetivos funcionais da organização, direcionando o esforço e o comportamento; fornecendo padrões a serem alcançados em relação ao objetivo; e em terceiro lugar, permitindo a congruência, alinhando metas entre as áreas funcionais de uma organização, controlando, dessa forma, atividades de grupos e indivíduos (Malmi \& Brown, 2008).

O sistema cibernético é concebido como um sistema de informação e apoio à decisão dependendo do seu uso. O controle cibernético permite a quantificação de um fenômeno, atividade ou sistema subjacente. Estabelece padrões de desempenho ou metas a serem cumpridas. Por meio de um processo de feedback permite a comparação do resultado das atividades com o padrão. Obtendo-se, assim, capacidade de modificar o comportamento do sistema ou atividades subjacentes (Malmi \& Brown, 2008).

Os sistemas de recompensa e compensação concentram-se em motivar e aumentar o desempenho de indivíduos e grupos dentro das organizações, alcançando a congruência entre seus objetivos e atividades e os da organização (Bonner \& Sprinkle, 2002). Os sistemas de controle administrativo direcionam o comportamento do funcionário de forma a especificar como as tarefas ou os comportamentos devem ser executados ou não (Malmi \& Brown, 2008). Por fim, a cultura organizacional é definida por Flamholtz et al. (1985) como um conjunto de valores, crenças e normas sociais que tendem a ser compartilhadas pelos membros da organização, influenciando seus pensamentos e ações. 
Figura 2: Sistema de Controle Gerencial na Perspectiva de Pacote.

\begin{tabular}{|c|c|c|c|c|c|c|}
\hline \multicolumn{7}{|c|}{ Controles Culturais } \\
\hline \multicolumn{2}{|c|}{ Crenças } & \multicolumn{3}{|c|}{ Valores } & \multicolumn{2}{|c|}{ Símbolos } \\
\hline \multicolumn{2}{|c|}{ Planejamento } & \multicolumn{4}{|c|}{ Controle Cibernético } & \multirow{2}{*}{$\begin{array}{l}\text { Remuneração e } \\
\text { recompensa }\end{array}$} \\
\hline $\begin{array}{l}\text { Planeja-mento } \\
\text { de longo prazo }\end{array}$ & Plano de ação & Orçamentos & $\begin{array}{l}\text { Sistema de mensuração } \\
\text { financeiro }\end{array}$ & $\begin{array}{l}\text { Sistema de mensuração } \\
\text { não financeiro }\end{array}$ & $\begin{array}{l}\text { Sistema híbrido } \\
\text { de mensuração }\end{array}$ & \\
\hline \multicolumn{7}{|c|}{ Controle Administrativo } \\
\hline \multicolumn{2}{|c|}{ Estrutura de Governança } & \multicolumn{3}{|c|}{ Estrutura Organizacional } & \multicolumn{2}{|c|}{ Políticas e Procedimentos } \\
\hline
\end{tabular}

Fonte: Malmi \& Brown (2008).

A Ambidestralidade é compreendida como a orientação que busca o equilíbrio entre inovação incremental e radical (Gupta, Smith \& Shalley, 2006). Considera-se que a inovação se destaca como um dos elementos cruciais na estratégia das organizações na busca de competitividade e sustentabilidade. Portanto, destaca-se a importância das organizações em dimensionar seu ambiente organizacional e buscar estruturas para capturar informações que tornem esse processo possível (Frezatti, Bido, Cruz, \& Machado, 2012). Nesse sentido, o ajuste entre o uso de ferramentas de controle gerencial com o nível de inovação abordado por Beuren e Oro (2014) indica que os controles formais contribuem para o processo de gestão da inovação, fornecendo informações necessárias ao processo de implementação de estratégias de diferenciação por meio de inovações de produtos.

A inovação é fundamental no desenvolvimento econômico, conforme destaca Shumpeter (1985) quando aborda a inovação como novas combinações, afirmando que o ajuste de recursos disponíveis pode resultar em algo diferente ou alterar a forma como fazê-lo. Dessa forma, a ênfase observada por Fargeberg (2005) resulta nos termos inovação de produto e inovação de processo. Ênfase também indicada nos tipos admissíveis de inovação, conforme definição de inovação da OCDE / Eurostat para fins de medição. Na 4 a edição do Manual de Oslo (2018), a inovação compreende inovações de produto, processo, organização e marketing.

A inovação no aspecto de estratégias tem como uma das abordagens orientações de exploration e exploitation. Uma empresa é considerada ambidestra quando estabelece um equilíbrio entre as habilidades de inovação incremental - exploitation e inovação radical - exploration (Dunkan, 1976; O'Reilly \& Tushman, 1996). A Ambidestralidade vem sendo abordada em pesquisas recentes como a capacidade de uma organização para atender às demandas mutuamente conflitantes de maneira efetiva (Birkinshaw, Zimmermann, \& Raisch, 2016). Para atingir tais objetivos, as organizações devem atender novas e atuais exigências, administrando simultaneamente as demandas de exploration e exploitation (Atuahene-Gima, 2005; Benner \& Tushman, 2003).

Conforme definido por March (1991), exploration é a orientação e a criação de novos conhecimentos que podem ser originados em ambientes externos das organizações - decorrentes de processos de parcerias, concorrência e clientes - ou internos - provenientes de áreas de pesquisa e desenvolvimento. As empresas voltadas para exploration apropriam-se de uma postura voltada a assumir riscos em razão da incerteza relativa ao sucesso e ao resultado a longo prazo (Jansen, Bosch, \& Volberda, 2006; Popadiuk, 2015).

O termo exploitation, entendido como inovação incremental, refere-se ao refinamento, escolhas e aperfeiçoamento de processos e rotinas (March, 1991). A fase de exploitation, abordada também por Gilsing (2003), é em geral caracterizada pela busca de crescimento econômico em um curto prazo, em razão da aprendizagem consolidada, utiliza-se de técnicas e rotinas já institucionalizadas, possibilitando, assim, os efeitos em maiores proporções. 
Uma das razões para o declínio das organizações é a incapacidade para mudar e inovar. Por esse motivo, diversos estudos organizacionais têm procurado explicar o papel direto dos diretores no desenvolvimento de uma orientação ambidestra (Lubatkin, Simsek, Ling, \& Veiga, 2006; Probst \& Raisch, 2005). A inovação é condição importante para a sobrevivência e manutenção das organizações. Ao se manter direcionada preferencialmente em exploitation, a empresa fica sujeita a um processo de obsolescência que, em médio ou longo prazo, pode levá-la a insolvência (Popadiuk, 2015).

\subsection{Contextualização e apresentação da hipótese}

Os resultados do trabalho de Revellino e Mouritsen (2009) mostraram que inovação passa por diferentes processos e que os controles se adaptam na medida em que o conjunto de controles, incluindo elementos de planejamento orçamentário, visão estratégica, satisfação do usuário, produtividade e metas de realização também se adapta conforme os processos de inovação organizacional amadurecem. Essa diversidade de controles adaptados à inovação ajuda a desenvolvê-la. Os controles são parte da inovação, não tendo somente o objetivo de torná-la transparente.

Bedford (2015), em pesquisa com dados coletados com gestores em 400 empresas, destacou que em empresas ambidestras as alavancas diagnósticas e interativas demonstram ter efeitos interdependentes no desempenho. Destaca também que algumas evidências sugerem que o uso combinado e equilibrado dessas alavancas contribui para gerar a tensão dinâmica necessária para o gerenciamento de modos de inovação.

O trabalho de Mackey e Deng (2016) examinou a associação entre uma estratégia de diferenciação e o surgimento de inovação usando um pacote de ferramentas de Sistemas de Controle Gerencial no nível operacional, por meio da aplicação de questionários aos estudantes dos programas de Executive Master of Bisiness Administration (EMBA) e Master in Business Administration (MBA) uma grande variedade de ex-alunos e estudantes de uma universidade pública nos Estados Unidos. Foram encontradas evidências de que as ferramentas de Sistema de Controle Gerencial abordadas são efetivas no aprimoramento da inovação.

O artigo de Antunes, Queiroz e Justino (2018) destaca os resultados que mostraram que os Sistemas de Controle Gerencial que se utilizam de indicadores não financeiros fornecem estratégias de inovação de produtos e processos. No entanto, os indicadores financeiros apenas beneficiam as estratégias de inovação relativas aos processos.

Os achados de Oliveira e Callado (2018), com o objetivo de avaliar as relações existentes entre os fatores contingenciais e as práticas de controle gerencial no âmbito de Organizações Não Governamentais (ONGs) brasileiras, foram de que fatores contingenciais externos e principalmente os internos influenciam os hábitos de controle gerencial utilizados pelos gestores das ONGs brasileiras.

Nesse contexto, salienta-se que pesquisas anteriores contribuem com as informações de que os controles contribuem para o processo de inovação (Revellino e Mouritsen, 2009; Bedford, 2015; Mackay e Deng, 2016; Antunes, Queiroz e Justino 2018). Alinhando-se com a hipótese desta pesquisa - H1 - que pretende comprovar que a utilização de Sistemas de Controles Gerenciais tem relação positiva com a Ambidestralidade, conforme destacado na Figura 3. 
Hipótese de Pesquisa: O uso de Sistema de Controle Gerencial tem relação positiva com a Ambidestralidade.

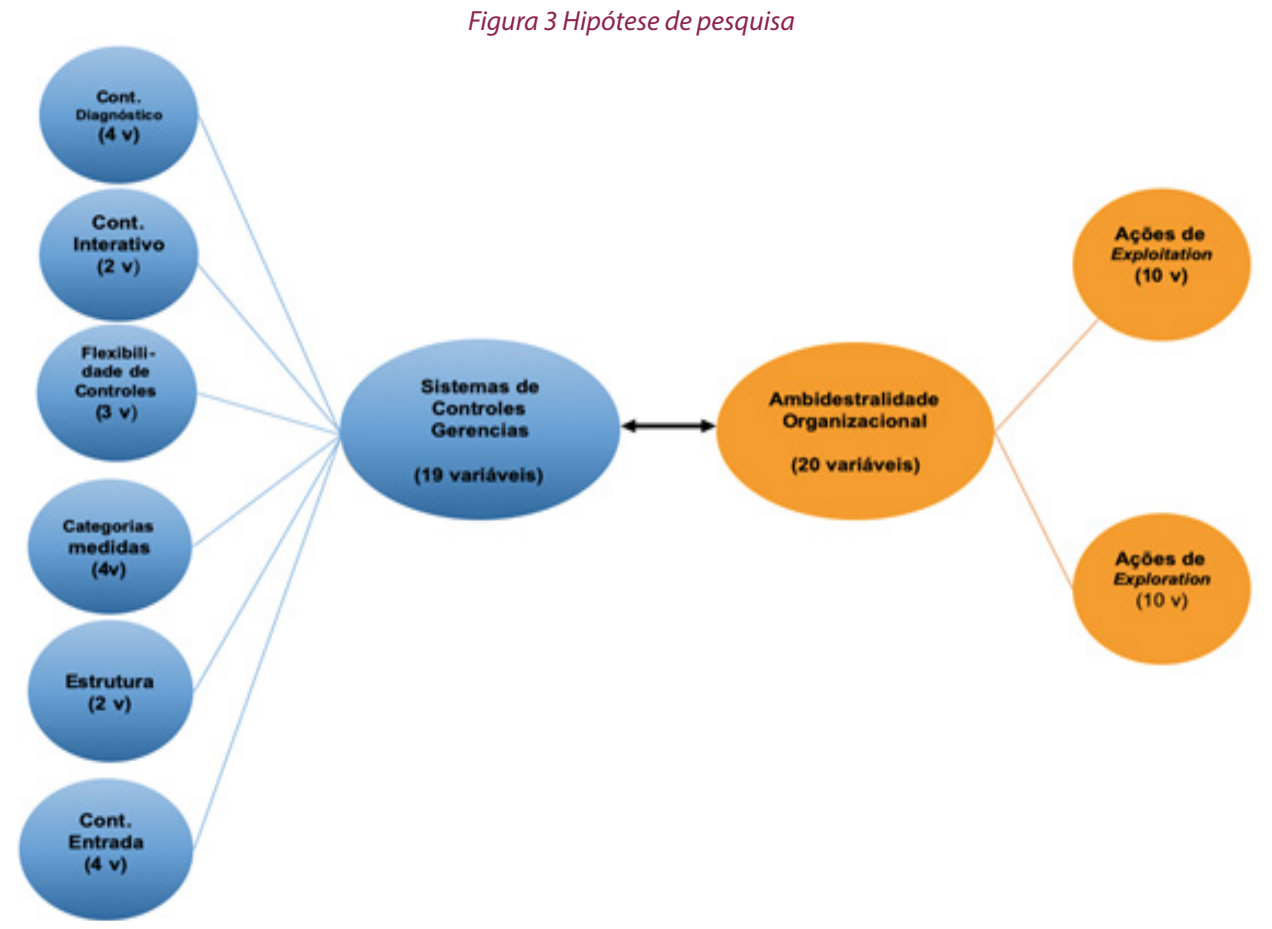

3 METODOLOGIA DA PESQUISA

A metodologia de pesquisa deste trabalho será apresentada da seguinte forma: caracterização da pesquisa, contextualização da hipótese de pesquisa, testes utilizados na pesquisa e aspectos operacionais.

Os Descritores do Planejamento da Pesquisa são apresentados na Tabela 1. O programa de pesquisa inclui o desenvolvimento da pesquisa a partir da elaboração da hipótese até a análise final dos dados (Cooper \& Schindler, 2016).

Tabela 1: Descritores do Planejamento da Pesquisa

\begin{tabular}{|c|c|}
\hline Categoria & Descritor \\
\hline Grau de cristalização da pesquisa & Estudo Exploratório \\
\hline Método de coleta de dados & Comunicação/instrumento autoadministrado \\
\hline $\begin{array}{c}\text { O poder do pesquisador de produzir efeitos nas variáveis que estão sendo } \\
\text { estudadas }\end{array}$ & Ex Post Facto \\
\hline Objeto de estudo & Descritivo \\
\hline A dimensão do tempo & Transversal \\
\hline Horizonte temporal investigado por constructo & Sistema de Controle Gerencial 2019 \\
\hline \multicolumn{2}{|l|}{ Ambidestralidade 2016 a 2019} \\
\hline As percepções dos participantes da atividade de pesquisa & Rotina real \\
\hline Sujeito da pesquisa - Respondente & Os gestores (presidentes) das APAEs \\
\hline Período da coleta de dados & Setembro/outubro 2019 \\
\hline População e amostra & $\begin{array}{l}\text { Classificada como censitária por adesão, contando com } \\
\text { 2.200 APAEs distribuídas em todo o território nacional. }\end{array}$ \\
\hline
\end{tabular}

Fonte: Adaptado Cooper \& Schindler (2016) 
A população e a amostra são compostas pelas Associações de Pais e Amigos dos Excepcionais (APAE), que se caracterizam por serem organizações sociais cujo objetivo principal é promover a atenção integral à pessoa com deficiência, prioritariamente aquela com deficiência intelectual e múltipla. Elas constituem uma rede que conta com mais de 700.000 pessoas atendidas, organizadas em 2.200 unidades presentes em todo o território nacional. A amostra foi censitária por adesão.

O pré-teste foi aplicado ao gestor da APAE de Anápolis por esta ter figurado, de 2017 a 2019, entre as 100 melhores ONGs do Brasil. A seleção realizada baseada em critérios sobre aspectos como: transparência e como as organizações avaliam seus resultados. O pré-teste também foi aplicado a estudantes e pesquisadores da área contábil. Com base nos trabalhos apresentados na Tabela 2, o questionário é constituído por afirmativas nas quais o respondente avalia a sua concordância por meio de uma escala tipo Likert de onze pontos. Os questionários foram enviados para endereços eletrônicos. Conforme Tabela 2, a estrutura do questionário foi dividida em 5 (cinco) blocos, neste trabalho serão utilizados 4 (blocos) distribuídos da seguinte forma:

Tabela 2: Estrutura Questionário

\begin{tabular}{|c|c|c|c|c|}
\hline Bloco & Constructo & Fatores & Quant. Variáveis & Periódico de origem \\
\hline 01 & Perfil respondente & & 04 & \\
\hline 02 & Perfil da instituição & & 08 & \\
\hline \multirow{6}{*}{03} & \multirow{3}{*}{$\begin{array}{c}\text { Sistema Controle } \\
\text { Gerencial }\end{array}$} & Controle Diagnóstico & 04 & \multirow{6}{*}{ Bedford, Malmi \& Sandelin (2016) } \\
\hline & & Controle Interativo & 02 & \\
\hline & & Flexibilidade de Controles & 04 & \\
\hline & & Categorias medidas de desempenho & 05 & \\
\hline & & Estrutura & 02 & \\
\hline & & Controle de Entrada & 04 & \\
\hline \multirow{2}{*}{04} & \multirow{2}{*}{$\begin{array}{l}\text { Ambidestralidade } \\
\text { Organizacional }\end{array}$} & Exploitation & 10 & \multirow{2}{*}{$\begin{array}{l}\text { Lubatkin, Simsek \& Veiga (2006) } \\
\text { Bedford, Bisbe \& Sweeney (2019) }\end{array}$} \\
\hline & & Exploration & 10 & \\
\hline
\end{tabular}

Fonte: Os autores (2020).

Com vistas ao maior entendimento desta pesquisa, e buscando contribuir com pesquisas futuras, as principais questões do instrumento de coleta de dados podem ser acessadas no apêndice 01 .

Quanto aos testes estatísticos, para conhecer a população foi utilizada estatística descritiva (média, desvio padrão). Para testar a hipótese de pesquisa foi utilizada a Correlação de Pearson e de forma suplementar testes de regressão linear simples.

\section{APRESENTAÇÃO DOS DADOS}

Foram coletadas um total de 446 respostas, representando um percentual $20,27 \%$ de taxa de respostas. Descartadas as respostas incompletas, o número de respostas foi para 269. Também foram excluídas as respostas com repetições de afirmativas, chegando-se então a um total de 227 respostas válidas, resultando em uma taxa de respostas de 10,32\%.

Com o objetivo de confirmar a consistência do instrumento de coleta de dados, e consequentemente confirmando a qualidade dos achados, a Figura 5 apresenta a avaliação da qualidade do instrumento de coleta. 
Figura 5 Avaliação da qualidade do instrumento de coleta.

\begin{tabular}{|c|c|c|c|c|c|}
\hline \multirow{3}{*}{$\begin{array}{c}\text { VALIDADE } \\
\text { Representações precisas } \\
\text { da característica que se } \\
\text { pretende medir }\end{array}$} & Teste & Descrição & \multicolumn{3}{|c|}{ Resultado } \\
\hline & Conteúdo & $\begin{array}{l}\text { Grau em que o instrumento } \\
\text { demonstre um domínio } \\
\text { específico de conteúdo do } \\
\text { que pretende medir. }\end{array}$ & \multicolumn{3}{|c|}{$\begin{array}{l}\text { O instrumento de coleta de dados recebeu diversas contribuições } \\
\text { na fase de pré-teste de pesquisadores dos referidos construtos. } \\
\text { Nessa fase, foram ajustados os itens: unidade de medida no } \\
\text { bloco de perfil da instituição, termos adequados ao ambiente das } \\
\text { organizaçôes pesquisadas referente ao construto de Sistemas de } \\
\text { Controles Gerencias. }\end{array}$} \\
\hline & Construto & $\begin{array}{l}\text { Grau em que um instrumento } \\
\text { de medidas se relacione } \\
\text { consistentemente com } \\
\text { outras medições derivadas da } \\
\text { mesma teoria. }\end{array}$ & \multicolumn{3}{|c|}{$\begin{array}{l}\text { Ao realizar a validade discriminante, foi evidenciado que os } \\
\text { fatores do construto Sistemas de Controles Gerenciais não } \\
\text { obtiveram uma correlação classificada como forte com os fatores } \\
\text { oriundos da Ambidestralidade Organizacional (exceto os fatores } \\
\text { Controle de Entrada com Exploitation } r=0,729) \text {, garantindo assim } \\
\text { a validade discriminante dos fatores. } \\
\text { Na validade convergente, foi identificado que todos os fatores de } \\
\text { cada construto se relacionam entre si. }\end{array}$} \\
\hline \multirow{5}{*}{$\begin{array}{l}\text { FIABILIDADE } \\
\text { (confiabilidade) } \\
\text { É Constância dos } \\
\text { resultados quando o } \\
\text { mesmo indivíduo ou } \\
\text { objeto é avaliando mais de } \\
\text { uma vez. }\end{array}$} & \multirow{5}{*}{$\begin{array}{l}\text { Alfa de } \\
\text { Cronbach }\end{array}$} & \multirow{5}{*}{$\begin{array}{l}\text { Mede a consistência interna } \\
\text { dos itens que compõem a } \\
\text { escala. }\end{array}$} & \multicolumn{3}{|c|}{$\begin{array}{c}\text { Os resultados dos testes de Alfa de Cronbach estão abaixo } \\
\text { apresentados: }\end{array}$} \\
\hline & & & Construto & $\mathrm{N}^{\circ}$ Itens & Coeficiente \\
\hline & & & $\begin{array}{c}\text { Ambidestralidade } \\
\text { Organizacional }\end{array}$ & 20 & 0,970 \\
\hline & & & Sistema de Controle Gerencial & 19 & 0,963 \\
\hline & & & \multicolumn{3}{|c|}{$\begin{array}{l}\text { Nota-se que o instrumento possui uma elevada confiabilidade } \\
\text { (acima de } 0,70 \text { ). }\end{array}$} \\
\hline \multirow{8}{*}{$\begin{array}{l}\text { SENSIBILIDADE } \\
\text { O objetivo é perceber se } \\
\text { a capacidade de medida } \\
\text { é capaz de discriminar } \\
\text { indivíduos estruturalmente } \\
\text { diferentes. }\end{array}$} & \multirow{8}{*}{$\begin{array}{l}\text { Testes de } \\
\text { distribuição }\end{array}$} & \multirow{4}{*}{$\begin{array}{c}\text { Curtose }(\mathrm{Ku}) \\
\text { Valor acima de } 7 \text { representa } \\
\text { graves problemas. }\end{array}$} & Construto & \multicolumn{2}{|c|}{ Construto Ku } \\
\hline & & & $\begin{array}{l}\text { Ambidestralidade } \\
\text { Organizacional }\end{array}$ & \multicolumn{2}{|c|}{2,518} \\
\hline & & & Sistemas de Controle Gerencial & \multicolumn{2}{|c|}{1,414} \\
\hline & & & \multicolumn{3}{|c|}{$\begin{array}{l}\text { Valor abaixo do valor crítico, demonstrando normalidade na } \\
\text { distribuição dos dados }\end{array}$} \\
\hline & & \multirow{4}{*}{$\begin{array}{c}\text { Assimetria }(\mathrm{Sk}) \\
\text { Valor acima de } 3 \text { representa } \\
\text { graves problemas. }\end{array}$} & Construto & \multicolumn{2}{|c|}{ Construto $\mathrm{Ku}$} \\
\hline & & & $\begin{array}{l}\text { Ambidestralidade } \\
\text { Organizacional }\end{array}$ & \multicolumn{2}{|c|}{$-1,480$} \\
\hline & & & Sistema de Controle Gerencial & \multicolumn{2}{|c|}{$-1,298$} \\
\hline & & & \multicolumn{3}{|c|}{$\begin{array}{l}\text { Valor abaixo do valor crítico, demonstrando normalidade na } \\
\text { distribuição dos dados. }\end{array}$} \\
\hline
\end{tabular}

Fonte: Adaptado de Martins e Domingues (2017), Favero e Belifiore (2017).

A partir da análise da qualidade do instrumento de coleta, a próxima seção analisará os perfis abordados no questionário.

\subsection{Perfil dos gestores investigados}

Quanto ao gênero dos participantes da pesquisa, destaca-se que 64\% são mulheres e 36\% são homens. A média etária dos gestores é de 54,63 anos com um desvio padrão de 12,379 anos. Quanto à formação educacional dos gestores, 37,5\% possuem o ensino médio completo; 34,4\% são graduados; 26,4\% são especialistas; $2,2 \%$ são mestres e 1,3\% são doutores. Ao questionar o tempo (em anos) de atuação como gestores da instituição, a média foi de 4,32 anos, com um desvio padrão de 3,437 anos.

\subsection{Características das organizações}

Participaram da pesquisa instituições de 20 estados. Quanto à localização geográfica, das 227 respostas válidas: 113 organizações (49,8\%) pertencem à região Sudeste; 55 (24,2\%) pertencem à região Sul; 21 (9,3\%) pertencem à região Norte; 20 (8,8\%) pertencem à região Nordeste; e 18 (7,9\%) pertencem à região Centro-Oeste. Quanto à idade de fundação das organizações, observou-se uma média de 30,45 anos com um desvio padrão de 13,071. A organização mais jovem possui 2 anos e a com maior tempo tem 60 anos.

O número médio de colaboradores atuando nessas organizações é de 26,78 empregados. A organização com o maior número possui 325 empregados e a com menor número possui 0 empregados. O número médio de voluntários regulares nas organizações é de 7,84 com um desvio padrão de 28 
voluntários; o número mínimo e máximo de voluntários é de 0 a 400 . O número médio de servidores cedidos pelo município ou pelo estado é de 13,09 servidores com um desvio padrão de 12,33, sendo o número mínimo e máximo de servidores 0 e 65, respectivamente.

\subsection{Nível de Ambidestralidade e Sistema de Controle Gerencial}

O construto Sistemas de Controles Gerenciais é composto pelos seguintes fatores: a) controle diagnóstico, b) controle interativo, c) flexibilidade de controles, d) categorias de medidas, e) estrutura (controles administrativos) ef) controle de entrada (controles culturais). O fator com a maior média foi o "fator de controle de entrada", acumulando 7,79 pontos e um desvio padrão de 2,27 pontos. Nesse fator, destaca-se a variável com as maiores médias:3.6.02. No fator estrutura destaca-se o fator de menor média: 6,04 com desvio padrão 2,81, e também se observa a variável com menor média V.3.5.02. Na Tabela 3 são apresentadas as médias dos fatores e das variáveis de forma analítica.

Tabela 3: Grau de Uso de Sistema de Controles Gerenciais

\begin{tabular}{|c|c|c|}
\hline Variáveis e Fatores & Média & Desvio Padrão \\
\hline V. 3.1.01 Identificar o alcance de metas que indicam a realização da estratégia atual & 6,70 & 3,185 \\
\hline V. 3.1.02 Monitorar o progresso em direção às metas & 6,85 & 3,096 \\
\hline V. 3.1.03 Fornecer informações para corrigir desvios de metas predefinidos & 7,01 & 3,172 \\
\hline V. 3.1.04 Revisar as principais áreas de desempenho & 7,30 & 3,110 \\
\hline FATOR CONTROLE DIAGNÓSTICO & 6,96 & 3,025 \\
\hline V. 3.2.01 Permitir o desafio contínuo e o debate de suposições e planos de ação com subordinados e pares & 7,15 & 3,019 \\
\hline V. 3.2.02 Incentivar o diálogo e o compartilhamento de informações com os subordinados & 7,54 & 3,024 \\
\hline FATOR CONTROLE INTERATIVO & 7,34 & 2,959 \\
\hline V. 3.3.01 Quão flexíveis são os objetivos de desempenho de subordinados depois de definidos: & 7,36 & 2,544 \\
\hline V. 3.3.02 Com que frequência os subordinados são consultados sobre o alcance de metas: & 7,95 & 2,611 \\
\hline V. 3.3.03 Até que ponto as explicações formais (por escrito) sobre o alcance de metas são exigidas dos subordinados: & 7,01 & 3,064 \\
\hline V. 3.3.04 Até que ponto as avaliações de subordinados são predominantemente baseadas no alcance de metas: & 6,93 & 2,979 \\
\hline FATOR FLEXIBILIDADE DOS CONTROLES & 7,31 & 2,474 \\
\hline V. 3.4.01 Satisfação de Usuários & 7,67 & 2,602 \\
\hline V. 3.4.02 Satisfação de Colaboradores & 7,43 & 2,721 \\
\hline V. 3.4.03 Eficiência do Processo operacional & 7,68 & 2,610 \\
\hline V. 3.4.04 Qualidade de Produtos e/ou Serviços & 8,01 & 2,493 \\
\hline FATOR CATEGORIAS DE MEDIDAS & 7,69 & 2,407 \\
\hline V. 3.5.01 Indique como as informações de controle são normalmente comunicadas em sua instituição: & 6,26 & 3,224 \\
\hline $\begin{array}{l}\text { V. 3.5.02 Quão extenso é o processo de recrutamento e seleção (busca por candidatos, uso de testes, entrevistas) para uma } \\
\text { posição gerencial: }\end{array}$ & 5,82 & 3,312 \\
\hline FATOR ESTRUTURA & 6,04 & 2,817 \\
\hline $\begin{array}{l}\text { V. 3.6.01 Quanta importância é dada à seleção de gerentes que tem atitudes e valores alinhados com a instituição, não } \\
\text { sendo considerado somente a competência? }\end{array}$ & 7,74 & 2,723 \\
\hline V. 3.6.02 Quanta importância é dada à formação e desenvolvimento de gerentes na sua instituição? & 7,93 & 2,508 \\
\hline $\begin{array}{l}\text { V. 3.6.03 Até que ponto os processos de treinamento e desenvolvimento são usados para reforçar os objetivos, } \\
\text { expectativas e normas da instituição? }\end{array}$ & 7,70 & 2,340 \\
\hline FATOR CONTROLE DE ENTRADA & 7,79 & 2,271 \\
\hline FATOR DE SEGUNDA ORGEM SISTEMAS DE CONTROLES GERENCIAIS & 7,19 & 2,213 \\
\hline
\end{tabular}

No construto Ambidestralidade Organizacional, o fator com a maior média foi o exploitation, acumulando 8,21 pontos e um desvio padrão de 1,919 pontos. Nesse fator, destacam-se as variáveis com as maiores médias: V 4.1.02 e V 4.1.03. No fator exploration, a variável com a menor média, inclusive do construto, foi 6,40 , totalizando 7,08 pontos. A média geral do construto foi de 7,65 pontos, com um desvio padrão de 2,052. Na Tabela 4 são apresentadas as médias das variáveis. 
Tabela 4: Grau de Ambidestralidade

\begin{tabular}{|c|c|c|}
\hline Variáveis e Fatores & Média & Desvio Padrão \\
\hline V 4.1.01 Busca melhorar gradualmente a qualidade de seus produtos e serviços & 8,29 & 2,110 \\
\hline V 4.1.02 Busca gradualmente reduzir os custos de seus produtos e serviços & 8,46 & 2,162 \\
\hline V 4.1.03 Busca aumentar gradualmente o grau de confiabilidade de seus produtos e serviços & 8,83 & 1,874 \\
\hline V 4.1.04 Procura ampliar os níveis de automação em suas operações & 7,76 & 2,520 \\
\hline V 4.1.05 Pesquisa frequentemente a satisfação dos usuários atuais & 7,69 & 2,699 \\
\hline $\begin{array}{l}\text { V 4.1.06 Desenvolve suas ofertas de produtos ou serviços, observando cuidadosamente as características dos seus atuais } \\
\text { usuários }\end{array}$ & 8,35 & 2,308 \\
\hline V 4.1.07 Busca estreitar e aprofundar as relações com seus usuários atuais & 8,62 & 2,092 \\
\hline V 4.1.08 Houve atualização dos conhecimentos e habilidades atuais para produtos ou serviços & 8,17 & 2,238 \\
\hline $\begin{array}{l}\text { V 4.1.09 Houve aprimoramento de habilidades em processos de desenvolvimento de produtos ou serviços nos quais a } \\
\text { empresa já possui experiência significativa }\end{array}$ & 7,88 & 2,328 \\
\hline $\begin{array}{l}\text { V 4.1.10 Houve fortalecimento do conhecimento e das habilidades para projetos que melhorem a eficiência das atividades } \\
\text { existentes de inovação de produtos ou serviços }\end{array}$ & 8,04 & 2,356 \\
\hline FATOR EXPLOITATION & 8,21 & 1,919 \\
\hline $\begin{array}{l}\text { V 4.2.01 Busca soluções tecnológicas pensando "fora da caixa", ou seja, fora dos limites da empresa, pesquisando } \\
\text { tecnologias diferentes das correntes? }\end{array}$ & 6,92 & 2,876 \\
\hline $\begin{array}{l}\text { V 4.2.02 Explica o desempenho da empresa em função da exploração de tecnologias inovadoras, ou seja, fundamenta seu } \\
\text { sucesso na habilidade em explorar novas tecnologias? }\end{array}$ & 6,73 & 2,841 \\
\hline V 4.2.03 Mantem foco na criação de novos produtos? & 6,51 & 3,103 \\
\hline V 4.2.04 Mantem foco na criação de novos serviços? & 7,14 & 2,892 \\
\hline V 4.2.05 Busca formas criativas e diferenciadas para satisfazer as necessidades de seus usuários? & 8,24 & 2,178 \\
\hline V 4.2.06 Utiliza novos produtos ou serviços para atuar em novos mercados? & 6,40 & 3,178 \\
\hline V 4.2.07 Utiliza a inovação para satisfazer as necessidades de seus usuários? & 7,72 & 2,575 \\
\hline V 4.2.08 Adquiriu-se habilidades inteiramente novas que são importantes para a inovação de produtos ou serviços? & 7,12 & 2,785 \\
\hline $\begin{array}{l}\text { V 4.2.09 Aprendeu-se habilidades e processos de desenvolvimento de produtos ou serviços totalmente novos para o seu } \\
\text { setor? }\end{array}$ & 6,89 & 2,876 \\
\hline $\begin{array}{l}\text { V 4.2.10 Houve fortalecimento das habilidades de inovação de produtos ou serviços em áreas onde não havia experiência } \\
\text { anterior? }\end{array}$ & 7,16 & 2,804 \\
\hline FATOR EXPLORATION & 7,08 & 2,442 \\
\hline FATOR DE SEGUNDA ORDEM AMBIDESTRALIDADE ORGANIZACIONAL & 7,65 & 2,052 \\
\hline
\end{tabular}

A partir dos dados obtidos, foi elaborada uma classificação quanto ao grau de Ambidestralidade Organizacional entre as APAEs pesquisadas. O critério utilizado foi o de que empresas com pontuação 0 a 5 entre os fatores exploitation e exploration são consideradas não inovadoras. As organizações com ações voltadas para exploitation alcançaram pontuação entre 5 e 10 para o fator exploitation e de 0 a 5 para ações de exploration; as organizações com ações voltadas para exploration alcançaram pontuação entre 05 e 10 para o fator exploration e de 0 a 5 para ações de exploitation; e por fim as organizações consideradas ambidestras alcançaram pontuação entre 5 e 10 para ambos os fatores. Essa classificação foi inspirada em Soares et al. (2018).

Quanto à distribuição das organizaç̃̃es entre as categorias inerentes à inovação, observa-se maior concentração das organizações classificadas como ambidestras: 179 representando (78,9\%); com ações voltadas a exploitation 29 (12,80\%); e com ações voltadas a exploration 5 (2,2 \%); as não inovadoras totalizam 14 (6,2\%).

Os dados da Tabela 5 demonstram que as empresas classificadas como não inovadoras possuem menores médias em todos os fatores relativos ao uso de controles gerenciais, em especial o fator controle de entrada média: 2,04. Já as empresas classificadas como ambidestras possuem as maiores médias em todos os fatores utilizados para medir o uso de Sistemas de Controles Gerenciais, em especial o fator controle de entrada de 8,43. 
Tabela 5 : Classificação de uso de Sistemas de Controle Gerenciais quanto ao grau de inovação organizacional

\begin{tabular}{|c|c|c|c|c|c|c|c|}
\hline \multicolumn{2}{|c|}{ Classificação de Inovação } & $\begin{array}{c}\text { Controle } \\
\text { Diagnóstico }\end{array}$ & $\begin{array}{c}\text { Controle } \\
\text { Interativo }\end{array}$ & $\begin{array}{c}\text { Flexibilidade } \\
\text { de Controles }\end{array}$ & $\begin{array}{c}\text { Categorias de } \\
\text { Medidas }\end{array}$ & $\begin{array}{c}\text { Estrutura } \\
\text { Controle de } \\
\text { Entrada }\end{array}$ \\
\hline Não Inovadora & Média & 2,55 & 2,86 & 3,34 & 3,07 & 2,04 & 2,93 \\
\hline & Desv. Padrão & 3,06 & 3,23 & 2,75 & 2,67 & 2,43 & 2,22 \\
\hline $\begin{array}{c}\text { Ações de } \\
\text { Exploitation }\end{array}$ & Média & 4,45 & 5,52 & 5,86 & 6,49 & 4,48 & 6,61 \\
\hline & Desv. Padrão & 3,26 & 3,49 & 2,47 & 2,61 & 2,42 & 2,61 \\
\hline $\begin{array}{c}\text { Ações de } \\
\text { Exploration }\end{array}$ & Média & 6,80 & 6,90 & 6,35 & 6,80 & 3,10 & 5,47 \\
\hline & Desv. Padrão & 3,41 & 3,09 & 3,28 & 3,30 & 3,09 & 2,02 \\
\hline $\begin{array}{c}\text { Organizações } \\
\text { Ambidestras }\end{array}$ & Média & 7,72 & 8,01 & 7,88 & 8,28 & 6,69 & 8,43 \\
\hline & Desv. Padrão & 2,42 & 2,38 & 2,02 & 1,80 & 2,49 & 1,54 \\
\hline
\end{tabular}

Em seguida foram abordados os testes de hipótese.

4.4 Testes de Hipóteses

Para avaliar a hipótese da pesquisa, foram utilizados testes de correlação de Pearson e regressão linear múltipla. É importante ressaltar que os comportamentos das variáveis e dos fatores atendem aos pressupostos da normalidade, conforme apresentado na Figura 6 e, dessa forma, foram utilizados testes paramétricos.

Nos resultados demonstrados na Figura 6, observa-se que os Sistemas de Controles Gerenciais possuem relacionamento positivo e significante com a Ambidestralidade Organizacional, confirmando-se assim a hipótese de pesquisa. Ao investigar o quanto os Sistemas de Controles Gerenciais são capazes de explicar a variação da Ambidestralidade Organizacional, identificou-se que esse percentual é de 59,80\% de variância explicada.

Analisando os índices por fatores, de forma analítica, identificou-se que o fator que mais explica a variação do construto Ambidestralidade Organizacional foi o controle de entrada, com uma relação positiva e significante de 0,72 pontos e uma capacidade de explicação de 52,60\%. Por outro lado, o fator com a menor capacidade explicativa, mas ainda sim significante, foi o fator estrutura com uma relação significante e positiva de 0,53 e uma capacidade explicativa de 28,70\%. 
Figura 6 Testes de Hipóteses

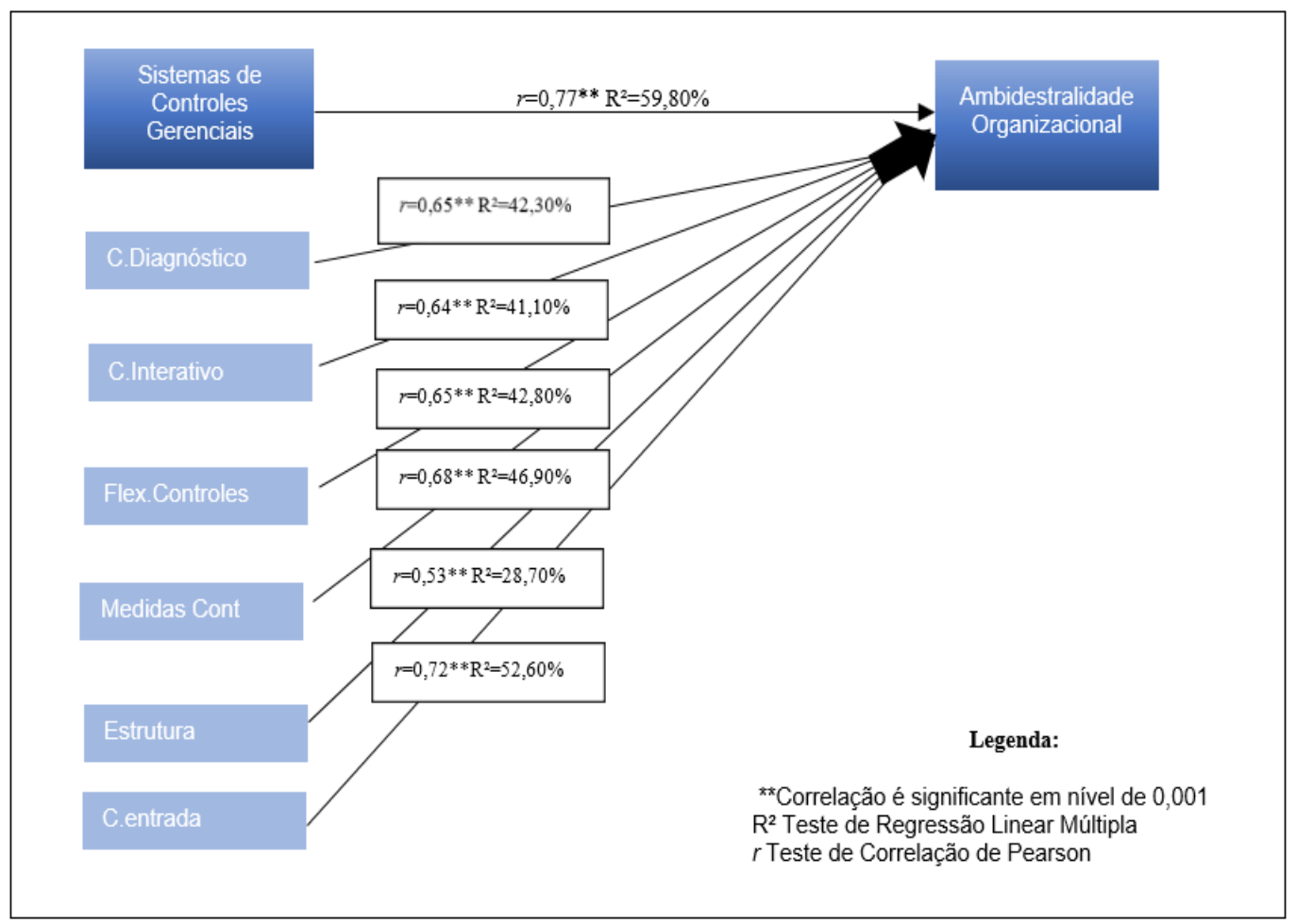

Portanto, comprova-se a hipótese de que o uso de Sistemas de Controles Gerenciais tem relação positiva e significativa com a Ambidestralidade. Tal comprovação foi realizada por meio do teste de correlação de Pearson com 0,77 e um fator de explicação de 59,80\%, comprovado através de regressão linear simples.

\section{DISCUSSÕES}

A análise da estatística descritiva dos dados evidencia uma maior tendência de empenho de esforços para alcançar a inovação a nível de exploitation em detrimento do nível de exploration, resultado indicado pelas médias de 8,21 e 7,08 respectivamente, conforme Figura 6 . O fato de a inclinação dos esforços ser maior para as ações de exploitation não difere de pesquisas anteriores, como os estudos aplicados ao setor bancário, abordados por Kuchpil (2019), e em que foram observadas médias para exploitation e exploration em 8,04 e 6,68 respectivamente.

O resultado deste trabalho também não diverge do resultado encontrado por Scandelari e Cunha (2013) no setor industrial de eletroeletrônicos em que foram encontradas médias de inclinação a exploitation de 4,06 e de exploration de 3,55. Os estudos de Soares, Reis, Cunha e Steiner Neto (2018) que trataram as instituições de ensino superior também corroboram o resultado encontrado neste estudo, no qual foram destacados os resultados de médias de 4,08 e 4,02 para exploitation e exploration respectivamente.

Com relação à capacidade de Ambidestralidade das organizações, foram encontradas neste trabalho uma média de organizações com competências de Ambidestralidade Organizacional de 7,65. O trabalho de Bedford, Bisbe e Sweeney (2019) encontrou uma média de 61,91 entre as empresas pesquisadas: empresas Irlandesas consideradas inovadoras; já no trabalho de Lubatkin, Simsek, Ling e Veiga (2006) 
foi encontrada uma média de 6,90 entre as pequenas e médias empresas que compunham a amostra de pesquisa.

A análise da estatística descritiva quanto ao uso de Sistemas de Controles Gerenciais nas organizações estudadas indica uma maior aplicação do uso de sistemas interativos em detrimento do uso de sistemas diagnósticos, com uma média de 7,34 e 6,96 respectivamente, mostrando um resultado diferente do encontrado por Bedford (2015) em empresas industriais nas quais foi observada uma média do uso de sistemas interativos de 4,78 e do uso de sistemas diagnósticos de 5,51.

A hipótese de pesquisa foi confirmada, pois os testes destacaram uma correlação $r=0,77$ a um $p=0,00$ entre os Sistemas de Controles Gerenciais e a Ambidestralidade, o índice de correlação é considerado forte (Dancey \& Reidy, 2006). Ao considerar o modelo de regressão é possível afirmar que $59,80 \%$ do comportamento da Ambidestralidade é explicado pelo comportamento do uso de Sistemas de Controles Gerenciais nas organizações estudadas. Esse é um valor considerado alto ao considerar a escala apresenta por Cohen (1977). E, destaca-se que o fator que mais contribuiu com o construto foi o "controle de entrada", com $r=0,72$ e um $\mathrm{R}^{2}=52,60 \%$, e o que menos contribuiu foi o fator "medidas de controle", com $r=0,53$ e um $R^{2}=28,70 \%$, ambos com significância a um nível de 0,001.

Os achados deste trabalho convergem com o trabalho de Revellino e Mouritsen (2009) no sentido de que os controles são parte da inovação, não sendo importante somente para torná-la transparente. Também vão de encontro com o trabalho de Bedford (2015) que destaca que o uso combinado e equilibrado de controles interativos e diagnósticos gera tensões dinâmicas que contribuem para que se possa existir modos de inovação. Em especial quando o fato de que a ênfase no uso do controle interativo está associada ao desempenho aprimorado das empresas envolvidas na inovação exploratória, mas não na explotação. Conforme verificado na Tabela 5, o controle interativo tem uma média maior que os controles diagnósticos em todos os níveis de classificação das organizações e se mostra crescente na medida em que a empresa se enquadra como explotatória, exploratória e ambidestra gradativamente.

Esses resultados também contribuem com o examinado por Mackey e Deng (2016) que encontraram evidências de que ferramentas abordadas de Sistemas de Controles Gerenciais são efetivas no aprimoramento da inovação. No mesmo sentido, o trabalho de Antunes, Queiroz e Justino (2018) apresenta que o uso de medidas financeiras e não financeiras contribui com estratégias de inovação de produtos e processos e de produtos. Este trabalho também está de acordo com o trabalho de Oliveira e Calado (2018) que demonstra que as organizações não governamentais se utilizam de Sistemas de Controles Gerenciais.

Conclui-se que após a demonstração dos testes e suas análises pode-se afirmar que estatisticamente existe relação positiva do uso de Sistemas de Controles Gerenciais com a Ambidestralidade, confirmando a hipótese abordada neste trabalho.

\section{CONSIDERAÇÕES FINAIS}

Conforme os dados obtidos, esta pesquisa confirma que há relação positiva e significante $\mathrm{r}=0,77$ entre o uso de Sistemas de Controles Gerenciais e a Ambidestralidade Organizacional. Os testes explicam a variação do comportamento ambidestro em organizações não governamentais pelo uso de Sistemas de Controles Gerenciais em 59,80\%.

Este estudo contribui teoricamente com a evidenciação do uso de Sistemas de Controles Gerenciais por organizações não governamentais e que estes contribuem com estratégias inovativas também no 
setor estudado, destacando competências de exploitation e exploration, além de demonstrar o nível de Ambidestralidade Organizacional nesse ambiente.

A contribuição prática baseia-se na apresentação de um panorama em organizações não governamentais do uso de Sistemas de Controles Gerencias e o grau de Ambidestralidade das organizações. Também contribui no aspecto prático com o aprimoramento dos mecanismos de controle, com o aperfeiçoamento de políticas de inovação, além da contribuição no treinamento dos gestores e elaboração de workshops e palestras, podendo ser um grande aliado nos programas de qualificação dos gestores das APAEs ou mesmo da Federação.

Quanto às limitações da pesquisa, destacam-se o fato de o questionário ter sido preenchido pelo gestor principal das APAEs, pois dessa forma a opinião sobre os assuntos abordados foi a partir do seu ponto de vista. Portanto, as interações comprovadas por este estudo não podem ser generalizadas. Outra limitação se refere ao fator da pesquisa se tratar de um corte temporal transversal, pois os fenômenos foram investigados em 2019.

A respeito de pesquisas futuras, sugere-se que sejam abordados outros tipos de teorias, aprimorando-se assim este modelo. Sob uma perspectiva quantitativa, sugere-se abordagens de estilos de liderança e estruturas de governança; sob uma perspectiva qualitativa, sugere-se abordagens sobre legitimidade do líder e legitimidade da organização perante a sociedade.

\section{AGRADECIMENTOS}

Agradecemos aos respondentes da pesquisa e, em especial, à Federação Nacional das APAEs por incentivar a pesquisa em organizações não governamentais no Brasil.

\section{REFERÊNCIAS}

Abernethy, M. A. \& Chua, W. F. (1996). A field study of control system "redesign": The impact of institutional processes on strategic choice. Contemporary Accounting Research, (13), 569-606.

Antunes, M. G., Queiroz, J. T., \& Justino, M. R. T. F. (2018). Role of Management Control Systems in Quality, Innovation and Organizational Performance in Portugal. SMES Companies. International Journal of Innovation and Technology Management, 15(2), 22 http://dx.doi.org/10.1142/S0219877018500141.

Atuahene-Gima, K. (2005). Resolving the capability-rigidity paradox in new product innovation. Journal of Marketing, 69(4), 61-83.

Bedford, D. S. (2015). Management control systems across different modes of innovation: Implications for firm performance. Management Accounting Research, 28, 12-30, http://dx.doi.org/10.1016/j. mar.2015.04.003.

Bedford, D. S., Malmi, T., \& Sandelin, M. (2016). Management control effectiveness and strategy: An empirical analysis of packages and systems, Accounting, Organizations and Society. 51 12-28. http:// dx.doi.org/10.1016/j.aos.2016.04.002.

Bedford, D. S., Bispe, J., \& Sweeney, B. (2019). Performance measurement systems as generators of cognitive conflict in ambidextrous firms. Accounting, Organizations and Society, 72, 21-37, https:// doi.org/10.1016/j.aos.2018.05.010. 
Beltrami, L. C., Gomes, S. M., \& Araújo, D. M. (2013). Sistemas de controle gerencial e desempenho corporativo: um estudo empírico em instituições de ensino superior. In: EnANPAD, 37, Anais eletrônicos. Rio de Janeiro, Brasil.

Benner, M. J., \& Tushman, M. L. (2003). Exploitation, exploration and process management: The productivity dilemma revisited. Academy of Management Review, 28(2), 238-257, https://doi.org/10.5465/ amr.2003.9416096.

Beuren, I. M., \& Oro, I. M. (2014). Relação entre estratégia de diferenciação e inovação, e sistemas de controle gerencial. Revista de Administração Contemporânea [S.I.], 18(3), 285-310, http://dx.doi.org/10.1590/1982-7849rac20141394.

Birkinshaw, J., Zimmermann, A., \& Raisch, S. (2016). How do firms adapt to discontinuous change? Bridging the dynamic capabilities and ambidexterity perspectives. California Management Review, 58(4), 36-58, http://doi:10.1525/cmr.2016.58.4.36.

Bonner, S. E., \& Sprinkle, G. B. (2002). The effects of monetary incentives on effort and task performance: Theories, evidence, and a framework for research. Accounting Organizations and Society 27(4/5), 303-345.

Cohen J. (1977). Statistical power analysis for the behavioral sciences. San Diego, CA: Academic Press.

Cooper, D. R., \& Schindler, P. S. (2016). Métodos de Pesquisa em Administração. (12a. ed.). Porto Alegre: AMGH.

Costa, E., Ramus, T., \& Andreaus, M. (2011). Accountability as a managerial tool in non-profit organizations: evidence from Italian CSVs. Voluntas, 22(3), 470-493.

Dancey, C. P.; Reidy, J. (2006). Estatística sem matemática para psicologia: usando SPSS para Windows. 3. ed. Porto Alegre: Artmed.

Davila, A., Foster, G., \& Oyon, D. (2009). Accounting and Control, Entrepreneurship and Innovation: Venturing into New Research Opportunities. European Accounting Review, 18(2), 281-311, https://doi. org/10.1080/09638180902731455.

Duncan, R. B. (1976). The ambidextrous organization: designing dual structures for innovation. In: R. H. Kilman, L. R. Pondy, D. Slevin (Eds.), The management of organization design: strategies and implementation (pp. 167-188). New York: University of Pittsburgh.

Fagerberg, J. (2005). Innovation: a guide to the literature. In: J. Fagerberg, D. C. Mowery, \& R. R.

Fávero, L. P., \& Belfiore, P. (2017). Análise de Dados, estatística e modelagem multivariadas com Excel, SPSS e Stata. (1 a. ed.). São Paulo: Atlas.

Flamholtz, E. G., Das, T. K., Tsui, A. S. (1985). Toward an integrative framework of organizational control. Accounting, Organizations and Society, 10:35-50.

Flamholtz, E. (1996). Effective organizational control: a framework, applications, and implications. European Management Journal, 14(6), 596-611.

Frezatti F., Bido, D. S. de, Cruz, A. P. C. da, \& Machado, M. J. C. de (2012). O papel do BSC na gestão da inovação. Anais do Encontro Nacional da Associação Nacional de Pós-Graduação e Pesquisa em Administração, Rio de Janeiro, RJ, Brasil, 36. 
Frezatti, F., Bido, D. S., Cruz, A. P. C., \& Machado, M. J. C. (2017). Impacts of Interactive and Diagnostic Control System Use on the Innovation Process. BAR - Brazilian Administration Review, 14. https://doi. org/10.1590/1807-7692bar2017160087.

Gilsing, C. B. (2003). Exploration, exploitation and co-evolution in innovation networks. Rotterdam: Erasmus University Rotterdam.

Gupta, A. K., Smith, K.G., \& Shalley, C. E. (2006). The interplay between exploration and exploitation. Academy of Management Journal, 49(4), 693-706.

Hall, B. (2010). The Financing of Innovative Firms. Review Of Economics And Institutions, 1(1). doi: http:// dx.doi.org/10.5202/rei.v1i1.4

Jansen, J. J. P., Van Den Bosh, F. A. J., \& Volberda, H. W. (2006). Exploratory Inovation, Exploitative Innovation and Performance: Effects of Organizational and Environmental Moderators Antecedents. Management Science, 52(11), 1661-1674.

Julian, D., \& Clapp, J. (2000). Outcomes based funding and the logic model: Mechanisms for coordinating human services at the local level. Evaluation and Program Planning, 23(2), 231-240.

Kruis, A., Speklé, R. F., \& Widener S. K. (2016). The Levers of Control Framework: An exploratory analysis of balance. Management Accounting Research, 32, 27-44. http://dx.doi.org/10.1016/j.mar.2015.12.002.

Kuchpil, I. (2019). Ambidestralidade Organizacional: Uma investigação de suas relações no desempenho de uma instituição financeira brasileira. Dissertação, Universidade Positivo, Curitiba. Paraná.

Lin, C., \& Chang, C. C. (2015). A patent-based study of the relationships among technological portfolio, ambidextrous innovation, and firm performance. Technology Analysis \& Strategic Management, 27(10), 1193- 1211. https://doi.org/10.1080/09537325.2015.1061119.

Lubatkin, M. H., Simsek, Z., Ling, Y., \& Veiga, J. F. (2006). Ambidexterity and performance in small- to mediumsized firms: The pivotal role of top management team behavioral integration. Journal of Management, 32, 646-672. https://doi:10.1177/0149206306290712.

Mackey, J. T., \& Deng, F. J. (2016). Examining the Role of Management Control Systems in the Creation of an Innovative Culture. International Journal of Innovation and Technology management, 13(3). doi: https://doi.org/10.1142/S0219877016400022.

Malmi, T., \& Brown, D. A. (2008). Management control systems as a package: Opportunities, challenges and research directions. Management Accounting Research, 19(4), 287-300. https://doi.org/10.1016/j. mar.2008.09.003

March, J. G. (1991) Exploration and exploitation in organizational learning. Organizational Science, 2(1), 71-87.

Martins, A. M., \& Domingues, O. (2017). Estatística Geral e Aplicada. (6a. ed.). São Paulo: Atlas S.A.

Merchant, K. A., \&Van der Stede, W. A. (2007). Management control systems: Performance measurement, evaluation and incentives (2a. ed.). Essex: Prentice Hall.

Moxham, C. (2009). Performance measurement: Examining the applicability of the existing body of knowledge to nonprofit organizations. International Journal of Operations and Production Management, 29, 740-763. https://doi.org/10.1108/01443570910971405. 
OCDE Organização para cooperação e desenvolvimento econômico (2018). Manual de Oslo: diretrizes para coleta e interpretação de dados sobre inovação (4a. ed.). Paris.

Oliveira, A. S., \& Callado, A. A. C. (2018). Fatores contingenciais e o controle gerencial: uma avaliação em organizações não governamentais (ongs) brasileiras. ASAA JOURNAL - Advances in Scientific and Applied Accounting, 11(1), 092-109. http://dx.doi.org/10.14392/asaa.2018110105.

Otley, D. T. (1980). The contingency theory of management accounting: achievement and prognosis. Accounting Organizations and Society, 4, 413-428.

Pletsch, C. S., Lavarda, C. E. F., \& Lavarda, R. A. B. (2016). Sistema de controle gerencial e sua contribuição para tensões dinâmicas. Enfoque: Reflexão Contábil, 35(3), 69-82. https://doi.org/10.4025/enfoque.v35i3.31344.

Popadiuk, S. (2015). Exploração, explotação e ambidestria: Inovação para a geração de valor. São Paulo: Mackenzie.

Probst, G., \& Raisch, S. (2005). Organizational crisis: The logic. Academy of Management Executive, 19(1), 90-105. https://doi.org/10.5465/ame.2005.15841958.

Raisch, S., Birkinshaw, J., Probst, G., \& Tushman, M. L. (2009). Organizational Ambidexterity: Balancing Exploitation and Exploration for Sustained Performance. Organization Science, 20(4), 685-695. https:// doi10.1287/orsc.1090.0428.

Ramadan, M. A., \& Borgonovi, E. (2015). Performance Measurement and Management in NonGovernmental Organizations. IOSR Journal of Business and Management, 17(2), 70-765. https://doi:10.9790/487X-17237076.

Revellino, S., \& Mouritsen, J. (2009). The multiplicity of controls and the making of innovation. European Accounting Review, 8(2), 341-369. https://doil:10.1080/09638180802681529.

Scandelari, V., \& Cunha, J. (2013). Ambidestralidade e desempenho socioambiental de empresas do setor eletroeletrônico. RAE-Revista De Administração de Empresas, 53(2), 183-198.

Schumpeter, J. A. (1985). A teoria do desenvolvimento econômico. São Paulo: Nova Cultural.

Simons, R. (1990). The role of management control systems in creating competitive advantage: new perspectives. Accounting, Organizations and Society, 15(112), 127-143.

Simons, R. (1995). Levers of Control. Harvard Business School Press, Boston.

Soares, J. L., dos Reis, D. R., da Cunha, J. C., \& Steiner Neto, P. J. (2018). Organizational Ambidexterity: A Study in Brazilian Higher Education Institutions. Journal of technology management \& innovation, 13(3), 36-46. http://dx.doi.org/10.4067/S0718-27242018000300036.

Teelken, C. (2008). The intricate implementation of performance measurement systems: Exploring developments in professionalservice organizations in the Dutch non-profit sector. International Review of Administrative Sciences, 74(4), 615-635,http://doi:10.1177/0020852308098471.

Tushman, M, O'Reilly, C. (1996). Ambidextrous Organizations: Managing Evolutionary and Revolutionary Change. California Management Review, 38(4), 8-29. 